\title{
LA FORMACIÓN DOCENTE INICIAL (FID) EN EDUCACIÓN PARVULARIA: DESATAR NUDOS.
}

INITIAL TEACHER TRAINING IN EARLY CHILDHOOD EDUCATION: TO UNTYING THE KNOTS.

\author{
Profesora Berta María Espinosa Vásquez \\ Especialista en Educación Parvularia \\ Directora Comité Chileno OMEP \\ Docente Carrera Educación Parvularia PUCV \\ Av. El Bosque 1290. Viña del Mar. Chile \\ berta.espinosa@ucv.cl
}

Resumen: Se presenta un análisis de algunos aspectos que constituyen trabas para el desarrollo autónomo de la formación docente inicial de educadores de párvulos, desde una perspectiva crítica hacia medidas de carácter instrumental y desde la lógica neoliberal de la universidad empresa.

Palabras clave: Acreditación, Prueba INICIA, Estándares Orientadores para la Formación en Educación Parvularia, Convenios de desempeño.

Abstract: An analysis of some aspects that constitute obstacles to the autonomous development of initial training of pre-school teachers is presented. This approach contains a critical perspective towards measures of instrumental character and from the neoliberal university business logic.

Key Words: Acreditation, INICIA test- Oriented standards for early childhood education - Performances covenant. 


\section{INTRODUCCIÓN}

Este texto constituye una reflexión que se realiza en el contexto en que se discute a nivel del senado de la república de Chile, el proyecto de ley que regulará el lucro y la selección y en el que se encuentran en consulta una serie de aspectos vinculados a la reforma educacional general como el proyecto de carrera docente y en particular la creación de la Subsecretaría de Educación Parvularia impulsada por el actual gobierno. Su propósito es iniciar una discusión sobre la temática de la FID, presentación realizada en el Seminario organizado por el comité chileno de la Organización Mundial de la Educación Preescolar (OMEP): Legislando sobre una educación de calidad para la primera infancia en chile: avances, desafíos y problemas realizado en la sede de la Cámara de Diputados, Santiago de Chile en el mes de agosto de 2014 y que fue expuesta en el panel: Percepción de la nueva institucionalidad y legislación desde la perspectiva profesional.

La acreditación oportunidad para ganar autonomía.

Si bien es cierto, que los procesos de acreditación de carreras de educación parvularia desde sus inicios a esta parte, han tenido como resultado avances en el promedio de años de acreditación y desde el punto de vista académico administrativo se ha logrado que las instancias superiores de las universidades, se ocupen de otorgar mejores condiciones para su funcionamiento de acuerdo a los planes de mejora levantados, no deja de ser preocupante que se siga visualizando la autoevaluación como un hito y no como proceso permanente, como un fin en sí misma y no como un medio para la mejora.

Las primeras acreditaciones, lograron que las carreras obtuvieran grados de autonomía suficiente para diseñar e implementar planes de estudios, de aumentar las plantas académicas y de conseguir mayores recursos para la docencia, sin embargo los procesos de autoevaluación de los programas siguen estando más atentos a los criterios del Consejo Nacional de 
Acreditación CNA, que a una política sólida de aseguramiento de la calidad en las instituciones de educación superior, el levantamiento de perfiles de egreso y modificaciones de los planes de estudios están sujetos a las condiciones que imponen antes los Proyectos de Mejoramiento de la Calidad de la Educación Superior MECESUP y en la actualidad los Convenios de desempeño y el Proyecto de Mejoramiento Institucional para la formación de docentes, que las universidades que se los adjudican diseñan.

Por otra parte, las plantas académicas de las carreras, no son suficientes para realizar la evaluación interna de los procesos, en el intento de conseguir mayor eficiencia y evidenciar en forma permanente en qué medida se alcanzan los propósitos declarados por el proyecto educativo de cada carrera, el apoyo institucional que se aporta si bien ha ido mejorando en cantidad, no necesariamente es de la calidad que requiere la implementación de procesos internos de seguimiento y monitoreo permanentes, así lo advertía la representante de un agencia de acreditación doña Judith Scharager (Agencia Qualitas) en el Seminario La Calidad de la Educación de La Infancia y el Sistema Evaluativo en Desarrollo para Cautelarla, organizado por el Comité Nacional de la Organización Mundial de la Educación Preescolar OMEP, en octubre de 2012, quien exponía que: "Los procesos de acreditación han provocado - en parte - que las organizaciones descansen en la regulación impuesta externamente, por sobre una autorregulación interna" y que ... "No se trata sólo de generar capacidades de respuesta administrativa a los requerimientos de regulación externos."

Medidas implementadas para regular la FID

Durante la anterior década, el Ministerio de Educación de Chile MINEDUC, ha ideado diversas acciones para mejorar la Formación Inicial Docente FID, cuyo carácter aislado y discontinuo ha generado una serie de inconvenientes a las instituciones formadoras y que han sido heredadas por la actual administración del país. Es así que tanto, los procesos de acreditación de carreras, como la prueba INICIA, los estándares orientadores para carreras 
de educación parvularia y la carrera docente se siguen observando por parte del mundo académico con no pocas tensiones y desconfianza, dado que su implementación sigue vigente y no se vislumbran grandes modificaciones que puedan augurar su mejoramiento.

Todas estas iniciativas constituyen mecanismos de control y regulación del estado al servicio de la consolidación de la lógica neoliberal de la educación como bien de consumo, mediante la figura de universidad empresa, la racionalización gerencial y la rentabilidad de los servicios.

Cualquier crítica al sistema ideado, se ha interpretado como una defensa corporativa y contra argumento sobre la calidad de la educación, postura tramposa, que debe evitarse, para acentuar la atención en lo que verdaderamente se requiere mejorar. Elevar los niveles de calidad de la FID requiere sin duda información, pero si la recogida de datos se realiza de una forma simplista, desde una perspectiva que concibe la realidad como asible, cuantificable y reducible a un parámetro, entonces se tiende a pensar que al no ser posible evaluar todo, entonces se evaluará lo medible, en el caso de la prueba inicia por ejemplo, se decide medir conocimientos pedagógicos y disciplinarios, la conclusión que se extrae no es cuánto conocimiento tiene una egresada, sino que si está bien o mal formada y si la institución en que se formó lo hizo mejor o peor.

La acreditación oportunidad para ganar autonomía.

Si bien es cierto, que los procesos de acreditación de carreras desde sus inicios a esta parte, han tenido como resultado avances en el promedio de años de acreditación y desde el punto de vista académico administrativo se ha logrado que las instancias superiores de las universidades, se ocupen de otorgar mejores condiciones para su funcionamiento de acuerdo a los planes de mejora levantados, no deja de ser preocupante que se siga visualizando la autoevaluación como un hito y no como proceso permanente, como un fin en si misma y no como un medio para la mejora. 
Las primeras acreditaciones, lograron que las carreras obtuvieran grados de autonomía suficiente para diseñar e implementar planes de estudios, de aumentar las plantas académicas y de conseguir mayores recursos para la docencia, sin embargo los procesos de autoevaluación de los programas siguen estando más atentos a los criterios del Consejo Nacional de Acreditación CNA, que a una política sólida de aseguramiento de la calidad en las instituciones de educación superior, el levantamiento de perfiles de egreso y modificaciones de los planes de estudios están sujetos a las condiciones que imponen antes los Proyectos de Mejoramiento de la Calidad de la Educación Superior MECESUP y en la actualidad los Convenios de desempeño y el Proyecto de Mejoramiento Institucional para la formación de docentes, que las universidades que se los adjudican diseñan.

Por otra parte, las plantas académicas de las carreras, no son suficientes para realizar la evaluación interna de los procesos, en el intento de conseguir mayor eficiencia y evidenciar en forma permanente en qué medida se alcanzan los propósitos declarados por el proyecto educativo de cada carrera, el apoyo institucional que se aporta si bien ha ido mejorando en cantidad, no necesariamente es de la calidad que requiere la implementación de procesos internos de seguimiento y monitoreo permanentes. Esto se ratifica con lo expresado por la representante de un agencia de acreditación doña Judith Scharager (Agencia Qualitas) quien en el Seminario organizado por el Comité Nacional de la Organización Mundial de la Educación Preescolar OMEP, en, exponía que "Los procesos de acreditación han provocado - en parte - que las organizaciones descansen en la regulación impuesta externamente, por sobre una autorregulación interna."

La prueba INICIA, como ranking.

La prueba INICIA La publicación de los resultados de la prueba INICIA, ha estado sometida a todo tipo de análisis, pero primordialmente se ha centrado en años anteriores en toda clase de juicios peyorativos sobre la formación en las carreras de pedagogía, lo que ha derivado en un ranking de instituciones 
formativas, suponiendo que los postulantes a este tipo de carreras y sus familias, deben conocer el puesto que ocupa la institución elegida, para asegurar que la inversión que se hará es la adecuada.

Ante esto, en agosto de 2013, el Consejo Nacional de Facultades de Educación de las Universidades pertenecientes al Consejo de Rectores de las Universidades Chilenas, frente al estilo de publicación de los resultados de la Prueba Inicia, señaló lamentar "el escaso rigor con que la información ha sido presentada a la opinión pública, toda vez que se trata de un proceso no sincronizado con otros en curso, como la innovación curricular de los programas de formación docente en desarrollo en las Universidades del CRUCH y cuyos efectos no son inmediatos".

Así también manifestaron que al ser no muestral, la aplicación, no sería posible arribar a conclusiones respecto del universo de los estudiantes de pedagogía, y advirtió respecto a la necesidad de "resguardar y fortalecer la profesión docente y por ello es necesario cuidar el tono comunicacional, a fin de no lesionar la imagen pública de ésta, en especial, la que imparten las Universidades representadas en este Consejo".

La prueba Inicia, surge muy bien intencionada, en un contexto de exigencia al Estado para que responda a la excesiva diversificación de las instituciones de formación de docentes cuya disparidad de calidades es bastante peligrosa, sin embargo, el centro de perfeccionamiento Experimentación e Investigaciones del CPEIP del MINEDUC organismo responsable de la implementación de INICIA exige a las Universidades para postular a los fondos de mejoramiento interno, de acuerdo al diagnóstico realizado por la propia institución, que se inscriba un 30\% de estudiantes inscritos para rendir la prueba y, por lo tanto la obliga a ceder, aunque la institución formadora no comparta esa lógica. En palabras simples, para tener acceso a dineros fiscales las instituciones de educación superior, deben comprometer ciertos resultados, de lo contrario deben devolver los montos asignados por el Ministerio de Educación MINEDUC. 
Los convenios de desempeño y la pérdida de autonomía

En los denominados Convenios de Desempeño, que vinieron a reemplazar a los Proyectos de Mejoramiento de la Calidad de la Educación Superior MECESUP, el rector de una universidad, debe restituir los fondos adjudicados. Esto no parece mal, toda vez que esos dineros provienen del aporte de la sociedad chilena en su conjunto, quienes cumplen a diario con el pago del impuesto al valor agregado IVA y anualmente con el impuesto a la renta.

El Convenio de Desempeño es un contrato entre el estado y una institución de educación superior mediante el que ésta se compromete a lograr desempeños notables que impliquen un significativo mejoramiento institucional, que no habría podido obtener sin el apoyo del estado. Este tipo de pacto, tienen asociados a su adjudicación ciertas condiciones que se negocian entre las universidades y el MINEDUC, una de estos requerimientos por ejemplo es el de elevar en forma gradual las exigencias de puntajes para el ingreso a las carreras de pedagogía, situación que se ha concretado a partir del año 2013, esta medida ha tenido como consecuencia un menor ingreso de postulantes a las universidades que se han adjudicado este tipo de convenios.

Resulta paradójico que una medida que organismos internacionales recomiendan como un factor que podría beneficiar la calidad de la formación, (UNESCO-OREALC, 2012) perjudique justamente a las instituciones de educación superior que hacen ingentes esfuerzos por mejorar la formación. ¿A dónde emigran las postulantes que obtienen puntajes menores a 530 o 550 puntos en la prueba de selección universitaria PSU? Justamente, las universidades privadas que poseen períodos de acreditación menores, son quienes captan a estas jóvenes y en un número no menor, con el agravante que no necesariamente estas instituciones de formación docente realizan esfuerzos suficientes para compensar las debilidades de estudiantes que ingresan, sea en periodos previos o iniciales de su formación, esta situación 
se puede apreciar con mayor nitidez en las regiones, en que las postulantes que no alcanzan a tener los puntajes solicitados por las universidades tradicionales se matriculan en las de carácter privado.

La concepción de evaluación que fundamenta las medidas

Pareciera ser que, intentando seria y bien intencionadamente mejorar los procesos de formación en los distintos niveles del sistema educativo, se hacen intentos desesperados, pero asentados sobre un enfoque que es muy discutible y que no ha sido suficientemente puesto en discusión o, que como todo lo perverso, se instala en el sistema rápidamente. Dada la influencia de los principios neoliberales, no parece extraño que los objetivos del sistema acreditación, prueba inicia estándares orientadores- se vinculen a ideas tales como la del valor agregado, la rendición de cuentas o el interés por informar a la demanda.

\section{A juicio de Santos Guerra}

“... la obsesión por la eficacia manifestada en los resultados da lugar a unas comparaciones entre Universidades, entre facultades, entre cursos, hartamente discutibles (MEC, 1989). Si no se parte de las mismas condiciones, si no se dispone de los mismos medios, si no se utilizan criterios adaptables y contrastados de evaluación, ¿cómo comparar lo incomparable? Esa comparación no es inocente, sino perversa. En el fondo tiende (y muchas veces consigue) a favorecer a las instituciones y a las personas más beneficiadas por la cultura, el dinero o el prestigio social. De esa forma, la evaluación se convierte en un instrumento de perpetuación y acentuación de las diferencias y de la injusticia" (1999:373)

En el sistema educativo de nuestro país se ha instalado una mirada de calidad desde una lógica empresarial, sólo de medición, y no de evaluación, como los docentes la entendemos. Es decir, un proceso de recogida de información con el propósito de tomar decisiones y no para tomar medidas punitivas, la evaluación tiene según Santos Guerra un trasfondo de mejora. En Chile como casi en ningún país del mundo, hay una fiebre por acreditar: la 
escuela, la institución formadora, los egresados; es decir, hay un marco en que la evaluación parece ya estar siendo algo siniestro.

La calidad es multifactorial

Las interrogantes que surgen acerca de la calidad de la FID en Educación Parvularia en Chile no se pueden responder únicamente solo desde el análisis de las propuestas curriculares. La problemática es multifactorial y requiere una mirada holística y sistémica que integre por ejemplo cómo realizan el ejercicio docente egresadas y egresados en el mundo laboral.

Una de las falencias que surgen desde el análisis de los procesos de autoevaluación con fines de acreditación de los programas de pregrado de educación parvularia es el escaso nivel de seguimiento que estas carreras realizan a sus egresadas, según Rojas y Falabella esto "obstaculiza una visión de más largo plazo sobre el impacto que puede tener esta formación en el ejercicio profesional de las educadoras" (2009:108)

Al mismo tiempo estas autoras plantean que: "En Chile existen experiencias de formación inicial interesantes en términos curriculares. La mayoría de los casos analizados han elaborado propuestas que ponen en el centro de la formación la reflexión y una identidad profesional centrada en el rol educador de las profesionales... están recién implementándose (llevan 3 o 4 años de vigencia), lo que impide realizar una evaluación de su efectividad de forma integral". (Ibid)

La existencia de una muy diversa calidad de la formación se ha tratado de regular por una parte con la obligatoriedad en la acreditación de las carreras de pedagogía y el levantamiento de estándares orientadores para la formación pedagógica y disciplinar, a esto se suma una medida que constituye parte del discurso reduccionista - los docentes no están 
preparados para enseñar materias que debieran dominar después de una formación universitaria- que es la habilitación, cuya condición es que se realice después de un período de ejercicio profesional de entre dos a tres años de ejercicio profesional.

\section{Autofinanciamiento y Autonomía}

En general las medidas adoptadas hasta el momento, atentan contra la autonomía de la universidad en general y de las carreras en particular, han alterado su tradicional misión formativa, y su vocación pública, entendida esta última como la defensa de un tipo de educación orientada hacia el bien común. La mayor parte de las universidades públicas, estatales y tradicionales se han visto subordinadas a la necesidad del autofinanciamiento con criterios de rentabilidad del conocimiento.

La lógica empresarial ha invadido el mundo de la educación superior imponiendo una suerte de neoliberalismo avanzado y variadas formas de capitalismo académico. Se exige que los perfiles de egreso se estructuren por competencias, dado el alto costo que implica esto y la dificultad para implementarlo por el bajo número de académicos de planta con que cuentan las instituciones, entonces se maquilla una especie de modelo bizarro denominado basado en competencias.

Una tensión que escapa a las propuestas formativas de las carreras de educación parvularia, pero que condiciona profundamente la formación, es el incremento del deterioro de las condiciones de empleo de las y los educadoras y educadores de párvulos. La oferta laboral es muy poco atractiva. La carrera vive un proceso de pérdida de heterogeneidad social y se focaliza cada vez más en un público que ingresa con vacíos o carencias cognitivas importantes, uno de cuyos factores es por ejemplo, la anteriormente mencionada forma en que las y los ministras/os de educación en conjunto con los medios, ha estado tratando los resultados de la prueba 
INICIA, es decir sin mayor profundidad en los análisis que se hacen para rankear a las instituciones formadoras.

Lo anterior se confirma con lo planteado por OREALC/UNESCO (2012) "Una carrera profesional atractiva supone remuneraciones adecuadas al momento del ingreso como asimismo que, a medida que el docente progresa, de acuerdo con su desempeño debidamente evaluado, pueda ver elevados sus ingresos económicos y acceder a nuevas oportunidades de desarrollo profesional" (p:139)

Sobre lo mismo, en su investigación Falabella y Rojas (2009) advierten: "Esta pérdida de mixtura social atenta contra los procesos pedagógicos de calidad. Frente a este escenario, fortalecer una propuesta formativa es doblemente difícil, pues es preciso invertir mucho tiempo y recursos en nivelar competencias escolares y, además, es contradictorio invitar a las estudiantes a adquirir una identidad profesional reflexiva si sus expectativas de sueldo se alejan bastante de la media que obtienen los profesionales de la educación o de otras áreas". (2009:110)

Súmese a lo anterior la nula posibilidad que tiene las docentes en ejercicio, para seguir perfeccionando la reflexión sobre su propia práctica, ejercicio que inician en la formación de pregrado, pero que se ve interrumpido al ingresar al campo laboral, puesto que los sostenedores - Municipalidades, Junta Nacional de Jardines Infantiles JUNJI, Fundación INTEGRA- , NO consideran tiempo destinado a este relevante aspecto del rol de una educadora, dentro del horario laboral.

Esta visión del problema, se ha ratificado en el documento Principios y Propuestas de El Plan Maestro cuando se reconoce que "cualquier esfuerzo por mejorar el desarrollo profesional docente debe contemplar una mejora previa de las condiciones laborales en que las profesoras y profesores ejercen su profesión. En la medida que no mejoren dichas condiciones, no podrá 
tampoco avanzarse en desarrollo profesional, porque ambas dimensiones están vinculadas". (2014:39)

Lo anteriormente expuesto, no significa desconocer que las instituciones formadoras deben hacer un esfuerzo por mejorar la calidad de sus procesos de formación, pero es probable que el impacto en la profesionalización de las educadoras de párvulos sea mayor y más efectivo cuando su estatus laboral adquiera una condición más profesional.

\section{Conclusión}

En síntesis, los nudos críticos de la FID son más que los nodos, en la actualidad, se requiere una Reforma Educacional con mayúscula, que contemple una política docente que apunte a solucionar los problemas vinculados a la problemática, para atraer y retener buenos docentes; que considere la carrera docente con al menos tres fases inicial, desarrollo y final, así también que contemple modalidades de promoción tanto horizontal como vertical; que supere la disociación entre carrera y desarrollo profesional, así como también, la tensión entre estructuras salariales comunes y remuneraciones diversificadas.

Del mismo modo no se podrá concretar la profesionalización docente, si no se vincula la FID con la formación continua, superando por un lado, la escasa relevancia y articulación de la oferta actual; el bajo impacto de las acciones hasta ahora emprendidas desde el CPEIP y por las instituciones del nivel JUNJI, INTEGRA y por otra parte, el desconocimiento de la heterogeneidad docente; la ampliación de oferta en forma desregulada; la falta de consideración del aprendizaje colaborativo en la realidad de las escuelas y jardines infantiles y una adecuada regulación y pertinencia de la oferta de postítulo y postgrados, se debe recordar que la mayor parte de estos no han sido acreditados.

Deshacer los nudos de la FID en educación parvularia implica una desafiante tarea que se logrará sólo con la generación de consensos, garantizada por 
una consulta amplia a todos los sectores involucrados y la participación activa no sólo de expertos de distintas disciplinas vinculadas a educación, sino de actores claves: estudiantes en formación, egresados de últimos años, docentes de aula con distintos años de ejercicio de la profesión y académicos formadores de formadores, sin la voz de los agentes principales del sistema, el nivel de compromiso y aceptación de las políticas que se implementen será muy bajo, casi nulo.

Bibliografía:

Falabella A. y M. T. Rojas, 2009

OREALC/UNESCO, 2012

OREALC/UNESCO, 2013

OREALC/UNESCO, 2013
Tensiones, debilidades y fortalezas en la formación de educadoras de párvulos en chile: un estudio de casos. En Revista electrónica diálogos educativos. Año $9, \mathrm{n}^{\circ} 18$, universidad metropolitana de ciencias de la educación. Santiago de Chile Antecedentes y Criterios para la Elaboración de Políticas Docentes en América Latina y el Caribe. Santiago de Chile.

Antecedentes y Criterios para la Elaboración de Políticas Docentes en América Latina y el Caribe. Profesores para una educación para todos. Proyecto estratégico regional sobre docentes. Santiago de Chile.

Estrategia Regional sobre Docentes i.e. Temas críticos para formular 
SANTOS GUERRA M., 1999

nuevas políticas docentes en América Latina y el Caribe. Santiago de Chile.

20 paradojas de la evaluación del alumnado en la Universidad española. Revista Electrónica Interuniversitaria de Formación del Profesorado. 2(1).

Consejo Consultivo. (2014)

El Plan Maestro. Diálogos para la Profesión Docente. Principios y Propuestas de El Plan Maestro. Disponible en: http://www.elplanmaestro.cl/wpcontent/uploads/2014/09/Propuestas-y Principios-de-El-Plan-Maestro.pdf 